\title{
Aortic Root Dissection In A Patient With Previous Aortic Valve Replacement Due To External Automated Chest Compression Device For Cardiac Arrest
}

Giacomo Bianchi ${ }^{1}$, Giovanni Concistrè ${ }^{1}$, Anees Al Jabri ${ }^{1}$, Cecilia Bianchi ${ }^{1}$, Elisa Barberi ${ }^{1}$, Sergio Berti ${ }^{1}$, Paolo Antonio Del Sarto ${ }^{1}$, and Marco Solinas ${ }^{1}$

${ }^{1}$ Stabilimento Ospedaliero di Massa Ospedale del Cuore G Pasquinucci

January 3, 2022

\begin{abstract}
Myocardial damage from external cardiac massage can occur with either manual massage or with an automatic external device. We report the case of a patient with an aortic valve bioprosthesis undergoing advanced resuscitation with an automated external device for out-of-hospital-cardiac arrest, in whom the prolonged compressions caused an aortic root dissection.
\end{abstract}

Aortic Root Dissection In A Patient With Previous Aortic Valve Replacement Due To External Automated Chest Compression Device For Cardiac Arrest

${ }^{1}$ Giacomo Bianchi, MD, PhD, ${ }^{1}$ Giovanni Concistrè, MD, ${ }^{2}$ Anees Al Jabri, MD,${ }^{3}$ Cecilia Bianchi, MD, ${ }^{3}$ Elisa Barberi, MD, ${ }^{2}$ Sergio Berti, MD,${ }^{3}$ Paolo Antonio Del Sarto, MD and ${ }^{1}$ Marco Solinas, MD

${ }^{1}$ Department of Adult Cardiac Surgery - Ospedale del Cuore - Fondazione Toscana "G. Monasterio" - Massa - ITALY

${ }^{2}$ Department of Interventional Cardiology - Ospedale del Cuore - Fondazione Toscana "G. Monasterio" Massa - ITALY

${ }^{3}$ Department of Anesthesiology and Critical Care - Ospedale del Cuore - Fondazione Toscana "G. Monasterio" - Massa - ITALY

Corresponding author:

Giacomo Bianchi, MD, PhD

Ospedale del Cuore - Fondazione Toscana "G. Monasterio" -

Via Aurelia Sud

54100, Massa - ITALY

email: gbianchi@ftgm.it

Short title: Aortic Root Dissection Due To External Chest Compression

Words: 1069

Keywords: ECMO; ECPR; LUCAS ${ }^{\mathrm{TM}}$; OHCA; STEMI; aortic root dissection; bioprosthesis

ABSTRACT 
Myocardial damage from external cardiac massage can occur with either manual massage or with an automatic external device. We report the case of a patient with an aortic valve bioprosthesis undergoing advanced resuscitation with an automated external device for out-of-hospital-cardiac arrest, in whom the prolonged compressions caused an aortic root dissection.

\section{CASE REPORT}

Given the nature of the case report, the hospital's IRB waived the consent; in addition, the treatment performed was not part of a clinical trial and therefore a registration was not necessary. A 72-year-old patient arrived at our center's shock room in refractory cardiac arrest; he was intubated, ventilated, and underwent automatic external cardiac massage using the LUCAS device. The underlying rhythm was lowvoltage ventricular fibrillation and $\mathrm{EtCO}_{2}$ of $23 \mathrm{mmHg}$. The no-flow time was less than 5 minutes and the low-flow time was 45 minutes. Given these parameters, the patient was deemed eligible for resuscitation with extracorporeal circulatory support (ECPR). Under echocardiographic guidance, the left femoral vein and artery were percutaneously incannulated using the Seldinger method with a 7 Fr introducer. An arterial blood gas (ABG) was performed demonstrating a $\mathrm{pH}$ of 7.1, $\mathrm{PaO}_{2}$ of $65 \mathrm{mmHg}, \mathrm{PaCO}_{2}$ of $25 \mathrm{mmHg}$, and a lactacidemia of $15 \mathrm{mmol} / \mathrm{L}$.

Thus, $100 \mathrm{IU} / \mathrm{kg}$ unfractionated heparin (UFH) was administered; the femoral artery was cannulated with Biomedicus (Medtronic, Minneapolis, USA) 17 Fr cannula and the femoral vein with Biomedicus (Medtronic, Minneapolis, USA) $25 \mathrm{Fr}$ cannula. The CardioHelp (Getinge, Goteborg, Sweden) system circuit was connected and support started progressively up to a flow of $3.5 \mathrm{~L} / \mathrm{min}$, adjusting $\mathrm{FiO}_{2}$ and gas blender to avoid hyperoxia, with a target $\mathrm{PaO}_{2}$ of $100 \mathrm{mmHg}$.

Support with Lucas was then discontinued. Approximately 5 minutes after reperfusion, an organized heart rhythm emerged, characterized by a third-degree atrioventricular block with a rate of $35 \mathrm{bpm}$.

A transthoracic echocardiogram was then performed and showed neither the presence of interventricular defect nor free wall rupture; moderate aortic insufficiency and the presence of pericardial effusion of approximately $2 \mathrm{~cm}$ was present.

The patient, after left radial artery catheter placement, was transferred to the catheterization lab for coronary angiography.

The interventional cardiologist established access from the right radial artery. After advancing guidance, an attempt to cannulate the left main was done, without success. An aortography was performed after exchanging the left Judkins catheter for a pig-tail. As can be seen in Figure 1, there is aneurysmal dilatation of the noncoronary sinus, associated with prosthesis rocking and paravalvular insufficiency. The left main was then incannulated: angiography showed that this was intact and that there was a subocclusive stenosis of the left anterior descending (LAD) artery followed by occlusion (Fugure 1B). A HeartTeam consultation deemed it appropriate to perform a disobstruction of the LAD in the first instance. The revascularization procedure, although with full VA-ECMO support, did not lead to reperfusion, resulting in a TIMI 1 (Figure 1C). On final angiography, root dissection was increased (Figure 1C) and on transesophageal echocardiogram the aortic root was completely disconnected, associated with massive paravalvular aortic insufficiency and tamponade. Due to the extent of the pathology, the previous surgery, the absence of reflow, the lack of cardiac contractility and the unknown neurological status, the Heart Team decided to suspend any further maneuver for futility.

\section{DISCUSSION}

Cardiac injuries from external cardiac massage is described in the literature, both in case of manual and automatic massage, with a percentage ranging from $2 \%$ to $8.5 \%$ and $7.9 \%$ to $12.5 \%$, respectively(1). Notable is the case report of a complete crush of a SAPIEN XT (Edwards, Irvine, CA - USA) after manual external cardiac massage(2). In general, a 2-fold risk of CPR-related injuries conferred by the use of a mechanical CPR device has been found(3). Forces developed during manual massage, in a study of paramedics, reached an average of $431 \mathrm{~N}(4)$, whereas automated devices can generate $530 \mathrm{~N}$ to $600 \mathrm{~N}$ at a frequency of 100 
compression per minute for essentially indefinite periods without any modulation from feedback of rib cage and internal organ resistance.

In the reported case, the presence of a stented valve represented an area of increased resistance that during cardiac massage by LUCAS ${ }^{\mathrm{TM}}$ produced traction on the surrounding tissues, tearing them. There is no relation between the event of cardiac arrest and the root dissection because it was determined by the subocclusive stenosis of the anterior descending artery and confirmed by the integration of the left main; therefore, it is not an extension of the dissection to the coronary arteries.

To our knowledge, this represents the first report of aortic root dissection resulting from automated external cardiac massage. A history of previous cardiac surgery with implantation of valve prostheses should raise suspicion of potential damage in case of application of automatic massage devices and potentially consider it a contraindication, moving towards manual massage.

Author contribution

Giacomo Bianchi MD, PhD: concept/design, drafting article, approval of article

Giovanni Concistré MD: drafting article, approval of article

Anees Al Jabri MD: drafting article, approval of article

Cecilia Bianchi MD: drafting article, approval of article

Elisa Barberi: drafting article, approval of article

Sergio Berti: approval of article

Paolo Antonio Del Sarto: approval of article

Marco Solinas: drafting article, approval of article

REFERENCES

1. Miller AC, Rosati SF, Suffredini AF, Schrump DS. A systematic review and pooled analysis of CPRassociated cardiovascular and thoracic injuries. Resuscitation. 2014 Jun;85(6):724-31.

2. Spangenberg T, Frerker C, Bader R, Schäfer U. Complete crush of a balloon-expandable bioprosthesis after prolonged cardiopulmonary resuscitation. Circ Cardiovasc Interv. 2013 Feb;6(1):e1-2.

3. Smekal D, Lindgren E, Sandler H, Johansson J, Rubertsson S. CPR-related injuries after manual or mechanical chest compressions with the LUCAS ${ }^{\mathrm{TM}}$ device: a multicentre study of victims after unsuccessful resuscitation. Resuscitation. 2014 Dec;85(12):1708-12.

4. Tomlinson AE, Nysaether J, Kramer-Johansen J, Steen PA, Dorph E. Compression force-depth relationship during out-of-hospital cardiopulmonary resuscitation. Resuscitation. 2007 Mar;72(3):364-70.

FIGURE LEGEND

FIGURE 1

A) Aortography showing the tear in the aortic root (arrow) with paravalvular leakage and the subocclusive stenosis of the LAD followed by occlusion. On the left-hand side, the venous drainage cannula (§) of the ECPR system. B) left coronary angiogram showing intact left main and LAD occlusion (*). C) Post-PCI angiogram with TIMI 1 final flow; aortic root laceration (\#). 


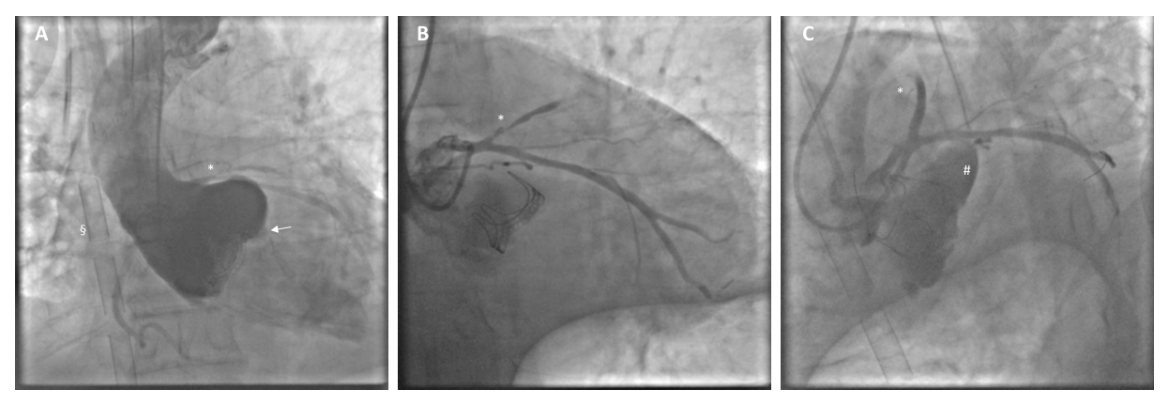

